\title{
Synthesis of Ketones and Ketimines via Organometallics and Secondary Amides
}<smiles>NC(N)=O</smiles>

$\mathrm{Tf}_{2} \mathrm{O}$ (1.1 equiv)

2-FPyr (1.1 equiv)

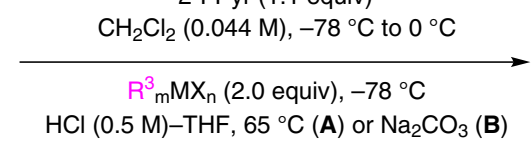

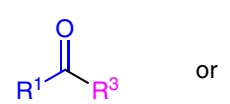

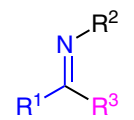

up to $98 \%$ yield (A)

up to $88 \%$ yield (B)

$\mathrm{R}^{1}=\mathrm{CO}_{2} \mathrm{Me}-, \mathrm{Br}-, \mathrm{CN}^{-}, \mathrm{NO}_{2}-$, azido-, $\mathrm{NMe}_{2^{-}}, \mathrm{HC}=\mathrm{CHCO}_{2} \mathrm{Et}-, \mathrm{N}=\mathrm{N}-\mathrm{Ph}-, \mathrm{C}(\mathrm{O}) \mathrm{Me}_{3}{ }^{-}, \mathrm{CHO}-, \mathrm{C}(\mathrm{O}) \mathrm{NEt}_{2}, \mathrm{C}(\mathrm{O}) \mathrm{N}(\mathrm{Me}) \mathrm{OMe}-$, $\mathrm{C}(\mathrm{O}) \mathrm{NHPh}, \mathrm{CH}_{2} \mathrm{Cl}$ - and $\mathrm{OAc}$-substituted $\mathrm{Ph}$, substituted pyridines, pyrimidines and thiophenes, $\mathrm{PhHC}=\mathrm{C}(\mathrm{Me}), \mathrm{Ph}\left(\mathrm{CH}_{2}\right)_{2}$, 4- $\mathrm{ClC}_{6} \mathrm{H}_{4} \mathrm{CH}_{2} \mathrm{O}, \mathrm{HC}(\mathrm{Me}) \mathrm{CH}_{2} \mathrm{OTBDOS}$, adamantyl

$\mathrm{R}^{2}=\mathrm{Cy}, \mathrm{CH}(i-\mathrm{Pr}) \mathrm{CH}_{2} \mathrm{OMe}, \mathrm{Bn}, \mathrm{CH}_{2} \mathrm{C}(\mathrm{H})=\mathrm{CH}_{2}$, chiral carbocycle

$\mathrm{R}^{3}=\mathrm{Et}$, decyl, s-Bu, $\left(\mathrm{CH}_{2}\right)_{2} \mathrm{Ph},\left(\mathrm{CH}_{2}\right)_{2} \mathrm{CH}=\mathrm{CH}_{2}, \mathrm{C}(\mathrm{Me})=\mathrm{CH}_{2}, \mathrm{Bn}, i-\mathrm{Pr}, \mathrm{Cy}$, Tol, anisyl, 4- $\mathrm{FC}_{6} \mathrm{H}_{4}, 4-\mathrm{ClC}_{6} \mathrm{H}_{4}$, furyl, 4- $\mathrm{NCC}_{6} \mathrm{H}_{4}$ 2-FPyr $=2$-fluoropyridine

$M=Z n, M g ; X=C l, B r ; m=1,2 ; n=0,1$

Selected examples:<smiles>CCOC(=O)C=Cc1ccc(C(=O)CC)cc1</smiles>

$84 \%$ yield<smiles>CCC(=O)c1cc(-c2nc(-c3ccccc3)cs2)c(C)nc1C(F)(F)F</smiles>

$99 \%$ yield<smiles>CCC(=O)/C(C)=C/c1ccccc1</smiles>

$95 \%$ yield

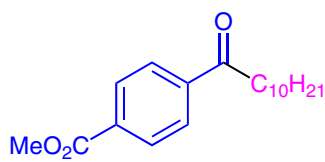

$86 \%$ yield<smiles>COc1ccc(C(=O)c2ccc(C=O)cc2)cc1</smiles>

$80 \%$ yield<smiles>CC(=O)c1ccc(C(=O)c2ccco2)cc1</smiles>

$68 \%$ yield<smiles>COc1ccc(C(=NC2CCCCC2)c2ccc(C)cc2)cc1</smiles>

$84 \%$ yield
Significance: A direct synthesis of ketones and ketimines using the nucleophilic addition of different zinc and magnesium reagents to secondary amides is disclosed. The corresponding ketones and ketimines are obtained in good to excellent yields.
Comment: Unlike the Weinreb amide, this protocol remarkably tolerates electrophilic and sensitive functionalities such as nitro, nitrile, methyl ester and $\mathrm{CF}_{3}$ groups. Furthermore, this methodology is characterized by high chemoselectivity, which is not guaranteed if conventional 1,2-addition reactions of organometallics to, for example, carboxylic chlorides are applied. 